\title{
Whole liver CT texture analysis to predict the development of colorectal liver metastases-A multicentre study
}

Citation for published version (APA):

Beckers, R. C. J., Lambregts, D. M. J., Schnerr, R. S., Maas, M., Rao, S-X., Kessels, A. G. H., Thywissen, T., Beets, G. L., Trebeschi, S., Houwers, J. B., Dejong, C. H., Verhoef, C., \& Beets-Tan, R. G. H. (2017). Whole liver CT texture analysis to predict the development of colorectal liver metastases-A multicentre study. European Journal of Radiology, 92, 64-71. https://doi.org/10.1016/j.ejrad.2017.04.019

Document status and date:

Published: 01/07/2017

DOI:

10.1016/j.ejrad.2017.04.019

Document Version:

Publisher's PDF, also known as Version of record

Document license:

Taverne

Please check the document version of this publication:

- A submitted manuscript is the version of the article upon submission and before peer-review. There can be important differences between the submitted version and the official published version of record.

People interested in the research are advised to contact the author for the final version of the publication, or visit the DOI to the publisher's website.

- The final author version and the galley proof are versions of the publication after peer review.

- The final published version features the final layout of the paper including the volume, issue and page numbers.

Link to publication

\footnotetext{
General rights rights.

- You may freely distribute the URL identifying the publication in the public portal. please follow below link for the End User Agreement:

www.umlib.nl/taverne-license

Take down policy

If you believe that this document breaches copyright please contact us at:

repository@maastrichtuniversity.nl

providing details and we will investigate your claim.
}

Copyright and moral rights for the publications made accessible in the public portal are retained by the authors and/or other copyright owners and it is a condition of accessing publications that users recognise and abide by the legal requirements associated with these

- Users may download and print one copy of any publication from the public portal for the purpose of private study or research.

- You may not further distribute the material or use it for any profit-making activity or commercial gain

If the publication is distributed under the terms of Article $25 \mathrm{fa}$ of the Dutch Copyright Act, indicated by the "Taverne" license above, 
Research paper

\title{
Whole liver CT texture analysis to predict the development of colorectal liver metastases-A multicentre study
}

\author{
Rianne C.J. Beckers ${ }^{\mathrm{a}, \mathrm{b}, \mathrm{c}, \mathrm{d}}$, Doenja M.J. Lambregts ${ }^{\mathrm{b}, *}$, Roald S. Schnerr ${ }^{\mathrm{c}}$, Monique Maas ${ }^{\mathrm{b}}$, \\ Sheng-Xiang Rao ${ }^{\mathrm{e}}$, Alfons G.H. Kessels ${ }^{\mathrm{f}}$, Thomas Thywissen ${ }^{\mathrm{c}}$, Geerard L. Beets ${ }^{\mathrm{a}, \mathrm{g}}$, \\ Stefano Trebeschi ${ }^{\mathrm{a}, \mathrm{b}}$, Janneke B. Houwers ${ }^{\mathrm{a}, \mathrm{c}}$, Cornelis H. Dejong ${ }^{\mathrm{d}, \mathrm{h}, \mathrm{i}}$, Cornelis Verhoef ${ }^{\mathrm{j}}$, \\ Regina G.H. Beets-Tan ${ }^{\mathrm{a}, \mathrm{b}}$ \\ a GROW School for Oncology and Developmental Biology, Maastricht University, P.O. Box 616, 6200 MD, The Netherlands \\ b Department of Radiology, The Netherlands Cancer Institute, P.O. Box 90203, 1006 BE Amsterdam, The Netherlands \\ ' Department of Radiology, Maastricht University Medical Center, P.O. Box 6200, 6202 AZ Maastricht, The Netherlands \\ d Department of Surgery, Maastricht University Medical Center, P.O. Box 6200, 6202 AZ Maastricht, The Netherlands \\ e Department of Radiology, Zhongshan Hospital, Fudan University, 180 Fenglin Road Shangai 200032, China \\ ${ }^{\mathrm{f}}$ Department of Clinical Epidemiology and Medical Technology Assessment, Maastricht University, P.O. Box 6200, 6202 AZ Maastricht, , The Netherlands \\ ${ }^{g}$ Department of Surgery, The Netherlands Cancer Institute, P.O. Box 90203, 1006 BE Amsterdam, The Netherlands \\ ${ }^{\text {h }}$ NUTRIM School for Nutrition and Translational Research in Metabolism, Maastricht University, P.O. Box 616, 6200 MD, The Netherlands \\ ${ }^{i}$ Department of Surgery, RWTH Universitätsklinikum Aachen, Pauwelsstraße 30, 52074 Aachen, Germany \\ ${ }^{j}$ Department of Surgical Oncology, Erasmus MC Cancer Institute, Groene Hilledijk 301, 3075 EA, Rotterdam, The Netherlands
}

\section{A R T I C L E I N F O}

\section{Keywords:}

Computed tomography

Texture analysis

Colorectal cancer

Liver metastases

Occult disease

Metachronous metastases

\begin{abstract}
A B S T R A C T
Objectives: CT texture analysis has shown promise to differentiate colorectal cancer patients with/without hepatic metastases.

Aim: To investigate whether whole-liver CT texture analysis can also predict the development of colorectal liver metastases.

Material and methods: Retrospective multicentre study $(\mathrm{n}=165)$. Three subgroups were assessed: patients [A] without metastases $(n=57)$, [B] with synchronous metastases $(n=54)$ and $[C]$ who developed metastases within $\leq 24$ months $(n=54)$. Whole-liver texture analysis was performed on primary staging CT. Mean greylevel intensity, entropy and uniformity were derived with different filters ( $\sigma 0.5-2.5)$. Univariable logistic regression (group A vs. B) identified potentially predictive parameters, which were tested in multivariable analyses to predict development of metastases (group A vs. C), including subgroup analyses for early ( $\leq 6$ months), intermediate (7-12 months) and late (13-24 months) metastases.

Results: Univariable analysis identified uniformity ( $\sigma 0.5)$, sex, tumour site, nodal stage and carcinoembryonic antigen as potential predictors. Uniformity remained a significant predictor in multivariable analysis to predict early metastases (OR 0.56). None of the parameters could predict intermediate/late metastases.

Conclusions: Whole-liver CT-texture analysis has potential to predict patients at risk of developing early liver metastases $\leq 6$ months, but is not robust enough to identify patients at risk of developing metastases at later stage.
\end{abstract}

\section{Introduction}

Survival in colorectal cancer (CRC) is influenced by several factors, including the local $\mathrm{T}$ - and $\mathrm{N}$-stage (tumour- and nodal stage), age and sex $[1,2]$. The most important factor, however, is the presence of metastases. Approximately $20 \%$ of patients have metastatic disease at time of diagnosis, with the liver being the most common site of metastases (77\%) [3-5]. Another 5\% of patients with primary nonmetastatic disease develop metachronous metastases within the first year, increasing to up to $15 \%$ at five-year follow-up [2]. Several

\footnotetext{
Abbreviations: AUC, area under the ROC curve; CEA, carcinoembryonic antigen; CRC, colorectal cancer; CT, computed tomography; E, entropy; LoG, Laplacian of Gaussian; M, mean

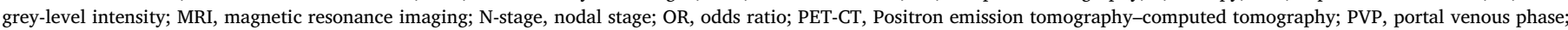
ROC, receiver operating characteristic; T-stage, tumour stage; U, uniformity; VOI, volume of interest

* Corresponding author.

E-mail address: d.lambregts@nki.nl (D.M.J. Lambregts).
} 
strategies have been explored to predict which patients are at risk of developing metachronous metastases. By identifying these patients, a different strategy with additional chemotherapy or intensified followup might be chosen. A known predictor for the development of colorectal metastases is an increased carcinoembryonic antigen (CEA) level [6]. Other factors are higher T-stage, lymph node metastases and male sex $[3,7,8]$. From an imaging perspective, a potentially promising tool is CT-texture analysis. Texture analysis refers to a mathematical approach to analyse variations in grey-level intensities within an image or region of interest to provide quantitative measures ('texture features') reflecting spatial heterogeneity [9-11]. Although several methods of texture analysis have been described in literature, the most commonly used technique is statistical-based texture analysis. The most basic form of statistical texture analysis involves first-order statistics, which focuses on the distribution of grey-level frequencies within an image, derived from the histogram of pixel intensities [11-13]. Commonly reported texture features include the 'mean intensity', 'entropy' and 'uniformity'. The mean intensity reflects the average pixel value or 'intensity' of a region of interest. Entropy is a measure that gives an indication of the irregularity of the grey-level distribution, while conversely the uniformity is indicative of its regularity. A higher entropy (and low uniformity) typically reflects a more "heterogeneous" distribution of pixels (and thus a more heterogeneous underlying tissue structure) while on the other hand a high uniformity is associated with a more "homogeneous" distribution of pixels (and therefore a more homogeneous tissue structure).

Single-centre studies have demonstrated that changes in the CTtexture of the liver (increase in entropy and decrease in uniformity) can be observed when the liver is affected by metastatic disease, thereby suggesting that these texture features have potential to differentiate between patients with and without colorectal liver metastases [10,14]. It has been suggested that these changes may be related to the presence of occult micro metastases throughout the liver and/or diffuse changes in hepatic perfusion caused by the presence of metastatic liver disease [15-19]. Hypothetically, similar changes - albeit to a lesser extent may already be present in an earlier phase, i.e. before the metastatic lesions become visible on morphological CT (computed tomography) assessment. If this were the case, CT-texture may also have potential as an imaging biomarker to predict upfront (at the time of primary staging) which patients who initially present without metastases are at risk of developing metastases at a later stage. This would be beneficial as CT is to date still the most widely used modality for staging of liver metastases and texture parameters can readily be obtained from routinely acquired clinical CT examinations.

The aim of this study was to evaluate in a multicentre study setting whether CT-texture analysis of the apparently non-diseased liver at the time of primary diagnosis has potential to predict patients at risk of developing liver metastases at a later stage.

\section{Material and methods}

\section{Patients}

This multicentre study analysed 165 patients (106 male, 59 female, median age 64 years, range 16-86 years) who were treated for colorectal cancer in one of three university hospitals between December 2006 and October 2013 (a time period selected based on adequate documentation of clinical patient data, availability of consistent quality imaging data and allowing for an adequate clinical follow-up period of at least 2 years). Patients routinely underwent contrast-enhanced liver or abdominal CT as part of their primary staging work-up. According to our country's national law, institutional review board approval and informed consent were not required for this retrospective study. Patients were divided into three \pm equally sized subgroups:
- Group A, the 'no metastases group', consisted of 57 patients who had no evidence of liver metastasis at primary staging, or during $\geq 24$ months of follow-up (established by means of clinical, laboratory (CEA) and imaging follow up).

- Group B, the 'synchronous metastases group', consisted of 54 patients who presented with synchronous liver metastases at the time of primary staging. The presence of metastases was confirmed by pathology (biopsy/surgery) in 17 patients, by corresponding positive findings on PET-CT in 7 patients, and by imaging follow-up in the other 30 patients who all had unresectable metastases, which were palliatively managed.

- Group C the 'metachronous metastases group', consisted of 54 patients who had no evidence of metastatic disease at primary staging but developed liver metastases (i.e. new and/or growing lesions on follow-up imaging) within 24 months after primary staging (median interval 12 months, range 2-24). In 21 patients these lesions were histopathologically proven to be colorectal liver metastases.

Inclusion criteria consisted of (a) histopathologically confirmed colorectal adenocarcinoma; (b) no evidence of extrahepatic metastatic sites on primary or follow-up imaging (CT, MRI and/or PET-CT); (c) availability of a primary staging CT including a portal venous phase; $(d)$ no history of previous liver surgery; (e) no history of previous systemic treatment (chemotherapy); ( $f$ ) no history of diffuse liver disease such as steatosis or cirrhosis; $(g)$ no history of diffuse hepatic metastases, as this would leave too little 'normal' liver parenchyma to perform whole-liver texture analysis. Clinical follow-up (after resection of the primary tumour) was performed according to routine clinical guidelines as advocated in the participating centres. This included routine (3-6 monthly) clinical examination, CEA testing and follow-up imaging with CT, MRI and/or FDG-PET when indicated.

\section{CT acquisition}

A contrast-enhanced CT was performed as part of the routine workup for CRC in all centres. All CT scans included a portal venous phase (PVP) scan of the liver and were acquired using different CT scanners (Philips Brilliance 64, Philips Medical Systems, Best, The Netherlands; Siemens Somatom Sensation 16, Somatom Sensation 64, Somatom Definition AS or Somatom Definition Flash, Siemens Healthcare, Erlangen, Germany; Toshiba Aquilion 64, Toshiba Medical Systems, Tokyo, Japan; GE Lightspeed VCT 64, GE Healthcare, Little Chalfont, United Kingdom). PVP images were routinely obtained with a tube voltage of $100-120 \mathrm{kVp}$. The contrast medium (Ultravist $300-370 \mathrm{mgI} /$ $\mathrm{ml}$; Iopromide, Bayer Healthcare, Berlin, Germany or Visipaque $320 \mathrm{mgI} / \mathrm{ml}$; Iopromide, GE Healthcare, Eindhoven, The Netherlands) was prewarmed to $37^{\circ} \mathrm{C}\left(99^{\circ} \mathrm{F}\right)$ and administered intravenously as a bolus injection of $100-150 \mathrm{ml}$ at a rate of $1,8-3,5 \mathrm{ml} / \mathrm{s}$, followed by a saline flush of 20-40 ml. The scan delay for the PVP was set at 70-80 s. Slice thickness was $5 \mathrm{~mm}$ in two of the three study centres. In the third center the original slice thickness was $3 \mathrm{~mm}$, which was reconstructed to $5 \mathrm{~mm}$ for assessment in this study.

\section{Image assessment}

CT images were transferred to an offline workstation for texture analyses and analysed using the open source software tool MRIcron [20]. An experienced reader (RCJB) manually traced the surface of the whole liver on the PVP images on each consecutive slice including all normal (apparently non-diseased) liver parenchyma, excluding the border of the liver, any visible lesions (any benign focal liver lesions or metastases), the caudate lobe (as this is often ill defined and difficult to discern from the vena cava), the inferior vena cava and large portal and hepatic veins to obtain volumes of interest (VOIs) of the whole liver volume, according to methods previously reported (Fig. 1) [10,14]. 


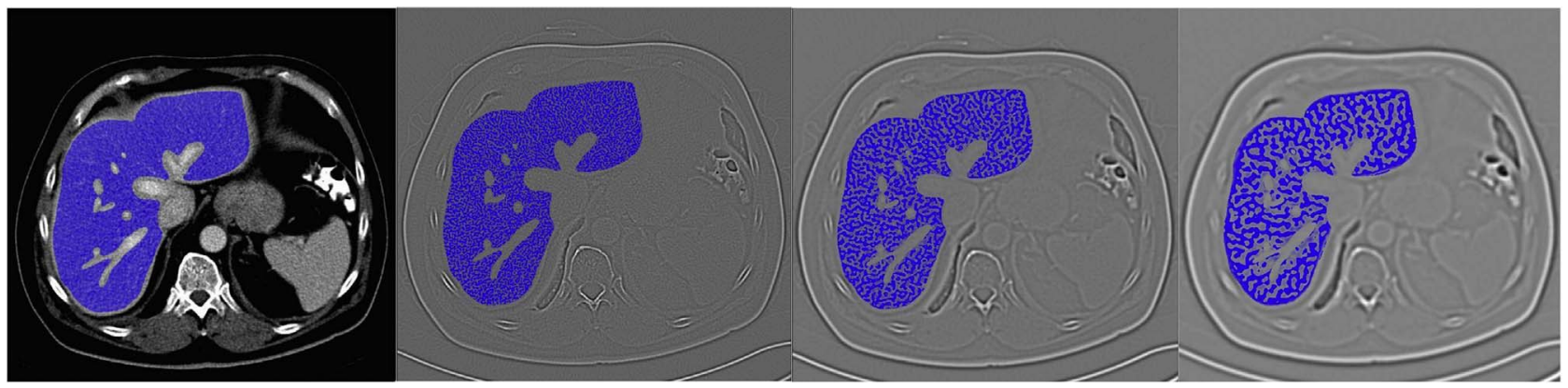

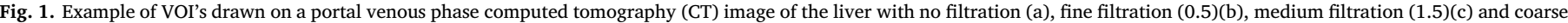
filtration $(2.5)(d)$. The border of the liver, any visible lesions, the inferior vena cava and large portal and hepatic veins were excluded.

Table 1

Baseline characteristics of the 165 study patients.

\begin{tabular}{|c|c|c|c|c|}
\hline & \multicolumn{3}{|l|}{ Group } & \multirow[b]{2}{*}{ Total } \\
\hline & $\mathrm{A}(\mathrm{N}=57)$ & $\mathrm{B}(\mathrm{N}=54)$ & $C(N=54)$ & \\
\hline \multicolumn{5}{|l|}{ Age (y) } \\
\hline Mean & 61 & 66 & 64 & 64 \\
\hline Range & $16-86$ & $22-85$ & $46-81$ & $16-86$ \\
\hline \multicolumn{5}{|l|}{ Sex } \\
\hline Male & $32(56.1)$ & $40(74.1)$ & $34(63.0)$ & $106(64.2)$ \\
\hline Female & 25 (43.9) & $14(25.9)$ & $20(37.0)$ & $59(35.8)$ \\
\hline \multicolumn{5}{|l|}{ Primary tumour site } \\
\hline Rectum & $34(59.6)$ & $19(35.2)$ & $24(44.4)$ & 77 (46.7) \\
\hline Colon & $23(40.4)$ & $35(64.8)$ & $30(55.6)$ & $88(53.3)$ \\
\hline \multicolumn{5}{|l|}{ Tumour stage" } \\
\hline $\mathrm{T} 1$ & $1(1.8)$ & 0 & 0 & $1(0.6)$ \\
\hline $\mathrm{T} 2$ & $8(14.0)$ & $2(3.7)$ & $3(5.6)$ & $13(7.9)$ \\
\hline $\mathrm{T} 3$ & $42(73.7)$ & $42(77.8)$ & $39(72.2)$ & $123(74.5)$ \\
\hline $\mathrm{T} 4$ & $6(10.5)$ & $1(1.9)$ & $12(22.2)$ & 19 (11.5) \\
\hline \multicolumn{5}{|l|}{ Nodal stage" } \\
\hline No & 25 (43.9) & $15(27.8)$ & $15(27.8)$ & $55(33.3)$ \\
\hline N1-2 & $32(56.1)$ & $30(55.6)$ & $39(72.2)$ & $101(61.2)$ \\
\hline \multicolumn{5}{|c|}{ Interval until metastases $(\mathrm{m})^{\dagger}$} \\
\hline & N/A & $\mathrm{N} / \mathrm{A}$ & $12[2-24]$ & $\mathrm{N} / \mathrm{A}$ \\
\hline \multicolumn{5}{|c|}{ Carcinoembryonic antigen (CEA) at the time of primary diagnosis } \\
\hline Normal $(<5 \mathrm{mcg} / \mathrm{L})$ & $24(42.1)$ & $7(13.0)$ & $20(37.0)$ & $51(30.9)$ \\
\hline Elevated ( $\geq 5 \mathrm{mcg} / \mathrm{L})$ & $18(31.6)$ & $39(72.2)$ & $25(46.3)$ & $82(49.7)$ \\
\hline
\end{tabular}

Note. Unless otherwise indicated, data are numbers of patients and data in parentheses are percentages.

Group A: patients without liver metastasis $(\mathrm{N}=27 / 11 / 19$ per centre $)$.

Group B: patients with synchronous liver metastasis at the time of primary staging $(\mathrm{N}=18 / 8 / 28$ per centre).

Group C: patients with liver metastasis occurring within 24 months after primary staging ( $\mathrm{N}=30 / 6 / 18$ per centre)

N/A: not applicable.

* Primary tumour/nodal stage based on histopathology for patients undergoing direct surgery and based on MRI for patients undergoing neo-adjuvant treatment.

${ }^{\dagger}$ Median interval between the primary staging CT and the detection of liver metastases.

\section{Texture analysis}

Texture analysis was performed using a dedicated script written in MATLAB (The MathWorks Inc., Natick, MA, 2000) by one of the authors (RSS), according to methods previously reported $[14,21]$. It comprised two main stages: (a) image filtration, followed by (b) quantification of texture. Fine image filtration highlights very small structures (in the order of 1-2 mm), while coarse image filtration highlights larger structures (in the order of $6 \mathrm{~mm}$ ). For image filtration, a Laplacian of Gaussian (LoG) band-pass filter was applied to the VOI using sigma ( $\sigma$ ) values of 0.5 (fine scale filtration), 1.5 (medium scale filtration) and 2.5 (coarse scale filtration). For each CT scan with a resolution different than $0.84 \mathrm{~mm}$, the value of $\sigma$ was modified to keep the physical size (in $\mathrm{mm}$ ) of the filter constant. The texture of the liver parenchyma was characterized by mean grey-level intensity (M), entropy (E) and uniformity (U). The mathematical analysis technique used for this study is described in detail in the Appendix.

\section{Statistical analysis}

Statistical analyses were performed using the Statistical Package for the Social Sciences (IBM Corp. Released 2012. IBM SPSS Statistics for Windows, Version 21.0. Armonk, NY: IBM Corp.) and Stata (StataCorp. 2009. Stata Statistical Software: Release 11. College Station, TX: StataCorp LP). Baseline patient characteristics were assessed using descriptive statistics. Further analysis comprised two steps:

- Step 1: univariable multilevel logistic regression analyses were performed to identify potentially predictive clinical and texture parameters $(P<0.1)$ to differentiate between group A (no metastases) and group B (synchronous metastases). Multilevel analyses per CT scanner ( $\mathrm{n}=10$ scanners, 3-4 different scanners per center) were performed to account for potential differences in scan protocol (e.g. contrast timing, $\mathrm{kVp}$ ) and $\mathrm{CT}$ software and hardware between scanners/centres.

- Step 2: multivariable multilevel logistic regression analysis was performed to test the predictive value of the clinical and texture parameters identified in step 1 to differentiate between patients without metastases (group A) and patients who later developed metastases (group C). CT scanner was chosen as level for the multilevel analysis. Results were separately analysed for patients who developed metastases within 6 months (early), between 7 and 12 months (intermediate) and between 13 and 24 months (late).

Receiver operating characteristics (ROC) curves were constructed to determine the diagnostic performance of the various clinical/texture features in predicting the development of liver metastases. The diagnostic performance of different combinations of parameters was assessed using predicted probabilities derived from the logistic regression as input variables. Differences in diagnostic performance between the different approaches were compared using the method described by Delong et al. [22].

\section{Results}

\section{Patient characteristics}

Patient characteristics are given in Table 1 . The primary tumour was located in the colon in 88 patients and in the rectum in the other 77 patients. Fifty-seven patients had no metastases (during a follow-up of at least 24 months after primary diagnosis); 54 patients had synchronous metastases; 11 patients developed metastases within 6 months, 20 within 7-12 months and 23 after 13-24 months. 
Table 2

CT texture parameters of the apparently non-diseased liver parenchyma.

\begin{tabular}{|c|c|c|c|c|c|c|}
\hline & $A(n=57)$ & $B(n=54)$ & $C(n=54)$ & $C^{1}(n=11)$ & $C^{2}(n=20)$ & $C^{3}(n=23)$ \\
\hline \multicolumn{7}{|c|}{ Mean grey-level intensity } \\
\hline unfiltered & $105.38 \pm 18.73$ & $103.09 \pm 17.34$ & $101.68 \pm 16.56$ & $100.61 \pm 12.67$ & $98.02 \pm 17.05$ & $105.37 \pm 17.59$ \\
\hline$\sigma=0.5$ & $0.001 \pm 0.07$ & $0.011 \pm 0.10$ & $-0.023 \pm 0.06$ & $-0.008 \pm 0.04$ & $-0.021 \pm 0.07$ & $-0.031 \pm 0.6$ \\
\hline$\sigma=1.5$ & $0.45 \pm 0.19$ & $0.50 \pm 0.23$ & $0.40 \pm 0.17$ & $0.45 \pm 0.18$ & $0.37 \pm 0.17$ & $0.40 \pm 0.18$ \\
\hline$\sigma=2.5$ & $1.42 \pm 0.44$ & $1.49 \pm 0.49$ & $1.34 \pm 0.44$ & $1.44 \pm 0.42$ & $1.23 \pm 0.38$ & $1.40 \pm 0.49$ \\
\hline \multicolumn{7}{|l|}{ Entropy } \\
\hline unfiltered & $5.69 \pm 0.26$ & $5.69 \pm 0.22$ & $5.65 \pm 0.34$ & $5.74 \pm 0.26$ & $5.68 \pm 0.38$ & $5.59 \pm 0.34$ \\
\hline$\sigma=0.5$ & $7.16 \pm 0.33$ & $7.04 \pm 0.37$ & $7.14 \pm 0.52$ & $7.36 \pm 0.23$ & $7.17 \pm 0.54$ & $7.00 \pm 0.58$ \\
\hline$\sigma=1.5$ & $6.87 \pm 0.35$ & $6.88 \pm 0.31$ & $6.78 \pm 0.38$ & $6.79 \pm 0.35$ & $6.83 \pm 0.44$ & $6.73 \pm 0.34$ \\
\hline$\sigma=2.5$ & $6.27 \pm 0.36$ & $6.35 \pm 0.30$ & $6.17 \pm 0.35$ & $6.17 \pm 0.35$ & $6.20 \pm 0.38$ & $6.15 \pm 0.32$ \\
\hline \multicolumn{7}{|l|}{ Uniformity } \\
\hline unfiltered & $23.24 \pm 4.06$ & $23.17 \pm 3.66$ & $24.08 \pm 5.54$ & $22.26 \pm 3.76$ & $23.76 \pm 5.88$ & $25.23 \pm 5.86$ \\
\hline$\sigma=0.5$ & $8.43 \pm 1.76$ & $9.37 \pm 3.00$ & $9.12 \pm 4.72$ & $7.29 \pm 1.14$ & $8.87 \pm 4.46$ & $10.21 \pm 5.73$ \\
\hline$\sigma=1.5$ & $10.47 \pm 2.54$ & $10.35 \pm 2.28$ & $11.16 \pm 2.66$ & $11.04 \pm 2.50$ & $10.89 \pm 3.03$ & $11.46 \pm 2.47$ \\
\hline$\sigma=2.5$ & $16.10 \pm 4.13$ & $15.21 \pm 3.32$ & $17.15 \pm 3.99$ & $17.18 \pm 3.87$ & $16.89 \pm 4.51$ & $17.36 \pm 3.71$ \\
\hline
\end{tabular}

Note: Data are the mean \pm standard deviation.

$\sigma:$ different image filtrations.

Group A: patients without liver metastasis.

Group B: patients with synchronous liver metastases at the time of primary staging.

Group C: patients with liver metastases occurring within 24 months following primary staging.

Group $C^{1}$ : early metastases ( $\leq 6$ months following primary staging).

Group $\mathrm{C}^{2}$ : intermediate metastases (7-12 months following primary staging).

Group $C^{3}$ : late metastases (13-24 months following primary staging).

\section{Step 1-Identification of potential predictive parameters}

The mean ( \pm standard deviation) of the texture parameters for the different subgroups are presented in Table 2. Detailed results of the univariable multilevel logistic regression analyses are presented in Table 3. There was no significant difference within the scanners $(\mathrm{P}>0.05)$. Of the texture parameters, uniformity at fine scale filtration $\left(\mathrm{U}_{0.5}\right)$ offered the best results to differentiate between patients

Table 3

Univariable multilevel logistic regression analysis to differentiate between group A and B.

\begin{tabular}{lll}
\hline Parameters & Odds Ratio & $P$ \\
\hline Mean grey-level intensity & & \\
$\quad$ unfiltered & $0.99(0.97-1.01)$ & 0.50 \\
$\sigma=0.5$ & $3.99(0.06-279.24)$ & 0.52 \\
$\sigma=1.5$ & $3.32(0.54-20.64)$ & 0.20 \\
$\sigma=2.5$ & $1.37(0.61-3.10)$ & 0.44 \\
Entropy & & \\
unfiltered & $0.99(0.21-4.60)$ & 0.99 \\
$\sigma=0.5$ & $0.32(0.10-1.05)$ & 0.06 \\
$\sigma=1.5$ & $1.14(0.36-3.60)$ & 0.82 \\
$\sigma=2.5$ & $2.00(0.64-6.24)$ & 0.23 \\
Uniformity & & \\
unfiltered & $1.00(0.90-1.10)$ & 0.93 \\
$\sigma=0.5$ & $1.21(0.99-1.46)$ & 0.06 \\
$\sigma=1.5$ & $0.98(0.84-1.14)$ & 0.79 \\
$\sigma=2.5$ & $0.94(0.85-1.04)$ & 0.21 \\
Age on diagnosis (y) ${ }^{\dagger}$ & $1.02(0.99-1.05)$ & 0.18 \\
Sex ${ }^{\dagger}$, & $0.45(0.20-1.00)$ & 0.05 \\
Primary tumour site ${ }^{\dagger}, \S$ & $2.72(1.26-5.88)$ & 0.01 \\
Tumour stage & $1.27(0.53-3.06)$ & 0.60 \\
Nodal stage & $1.52(0.96-2.39)$ & 0.07 \\
Node positive/Node negative & $1.56(0.69-3.52)$ & 0.28 \\
CEA level (mcg/L) & $1.05(1.02-1.09)$ & 0.00 \\
\hline
\end{tabular}

Note. Data in parentheses are $95 \%$ confidence intervals.

Group A: patients without liver metastasis.

Group B: patients with synchronous liver metastases at the time of primary staging.

* Since entropy and uniformity are correlated parameters, only uniformity was selected for multivariable logistic regression analysis because of the smaller confidence interval.

${ }^{\dagger}$ Parameters with $P<0.2$ were selected for multivariable logistic regression analysis.

* Male (vs. female).

$\S$ Location in rectum (vs. colon). without (group A) and patients with synchronous metastases (group B). In addition, nodal status, primary tumour site, sex and CEA were identified as potentially predictive clinical parameters.

\section{Step 2: prediction of metachronous metastases}

Table 4 shows the results of the multivariable logistic regression of the different parameters identified in step 1 to discriminate between group A (no metastases) and C (metachronous metastases). For the whole group of patients with metachronous disease neither texture analysis $\left(\mathrm{U}_{0.5}\right)$, nor any of the clinical parameters showed significant predictive value (OR $0.52-1.67, P=0.16-0.44$ ). In the sub-analysis of patients who developed early metastases (within 6 months) $\mathrm{U}_{0.5}$ resulted in an OR of $0.56(P=0.05)$. Representative imaging examples of patients from the no metastases and early metastases group are provided in Fig. 2. Nodal stage in this subgroup resulted in an OR of $3.19(P=0.06)$.

\section{Diagnostic performance to predict metachronous metastases}

ROC-curves illustrating the diagnostic performance of texture and clinical parameters to predict the development of liver metastases are provided in Fig. 3. For the whole group of patients with metachronous disease, AUCs to predict the development of metastases ranged between 0.51 and 0.61 for (combinations of) $\mathrm{U}_{0.5}$, and the clinical parameters CEA and nodal stage. For prediction of early metastases (occurring within six months), the AUC was 0.74 for $\mathrm{U}_{0.5}$ versus AUC 0.70 for CEA $+\mathrm{N}$-stage $(P=0.72)$. Combining $\mathrm{U}_{0.5}$ with CEA $+\mathrm{N}$-stage resulted in an AUC of 0.78, which was not a significant improvement compared to either $\mathrm{U}_{0.5}(P=0.53)$ or N-stage + CEA $(P=0.08)$.

\section{Discussion}

The aim of our study was to evaluate in a multicentre setting whether CT-texture analysis of the apparently non-diseased liver at the time of primary diagnosis has the potential to predict upfront which patients who initially present without metastases are at risk of developing liver metastases at later stage. Our results suggest that texture analysis has some promise to differentiate patients that will develop liver metastases early ( $\leq 6$ months) after initial diagnosis, but is not 
Table 4

Multilevel multivariable logistic regression analysis to differentiate between group A and C.

\begin{tabular}{|c|c|c|c|c|c|c|c|c|}
\hline \multirow[t]{2}{*}{ Parameters } & \multicolumn{2}{|l|}{ A vs. C } & \multicolumn{2}{|l|}{ A vs. $C^{1}(n=11)$} & \multicolumn{2}{|l|}{ A vs. $C^{2}(n=20)$} & \multicolumn{2}{|l|}{ A vs. $C^{3}(n=23)$} \\
\hline & Odds Ratio & $P$ & Odds Ratio & $P$ & Odds Ratio & $P$ & Odds Ratio & $P$ \\
\hline Uniformity $\sigma=0.5$ & $1.08(0.92-1.26)$ & 0.35 & $0.56(0.31-1.00)$ & 0.05 & $1.04(0.85-1.28)$ & 0.68 & $1.20(0.97-1.47)$ & 0.09 \\
\hline Nodal stage & $1.50(0.85-2.68)$ & 0.16 & $3.19(0.96-10.66)$ & 0.06 & $2.02(0.93-4.40)$ & 0.08 & $0.87(0.42-1.80)$ & 0.70 \\
\hline Primary tumour site ${ }^{*}$ & $1.67(0.68-4.10)$ & 0.26 & $4.18(0.70-25.02)$ & 0.12 & $1.95(0.58-6.60)$ & 0.28 & $1.49(0.46-4.81)$ & 0.50 \\
\hline $\mathrm{CEA}(\mathrm{mcg} / \mathrm{L})$ & $1.01(0.98-1.05)$ & 0.44 & $1.02(0.98-1.06)$ & 0.41 & $1.02(0.98-1.06)$ & 0.37 & $1.00(0.94-1.06)$ & 0.93 \\
\hline Sex ${ }^{\dagger}$ & $0.52(0.21-1.31)$ & 0.17 & $0.63(0.12-3.38)$ & 0.59 & $0.69(0.19-2.49)$ & 0.57 & $0.45(0.13-1.58)$ & 0.21 \\
\hline
\end{tabular}

Note. Data in parentheses are $95 \%$ confidence intervals.

Group A: patients without liver metastases.

Group C: with liver metastases occurring within 24 months following primary staging.

Group $\mathrm{C}^{1}$ : early metastases ( $\leq 6$ months following primary staging)

Group $C^{2}$ : intermediate metastases (7-12 months following primary staging).

Group $\mathrm{C}^{3}$ : late metastases (13-24 months following primary staging).

* Location in rectum (vs. colon)

${ }^{\dagger}$ Male (vs. female).

robust enough to identify patients at risk to develop metastases after a longer interval (within 7-24 months).

Our findings are in line with those of previous single-center studies that reported that changes in the overall texture of the liver parenchyma can be observed when metastatic disease is present within the liver. Both Rao et al. [14] and Ganeshan et al. [10] reported significant differences in CT-texture between patients with and without liver metastases. Rao et al. [14] also reported a similar subtle change in texture in patients who had no metastases at the time of primary diagnosis but later developed metachronous liver metastases, but the number of patients with metachronous disease in their cohort was too small $(n=4)$ to draw any meaningful conclusions. Our current multicentre study follows up on these previous works and confirms that - at least to predict metastases occurring within 6 months - texture analysis may be of added value. In ROC-curve analysis there was a trend towards improved results compared to more well-known clinical markers (CEA + N-stage) with an AUC of 0.78 for texture + clinical markers versus an AUC of 0.70 for only clinical markers, albeit not
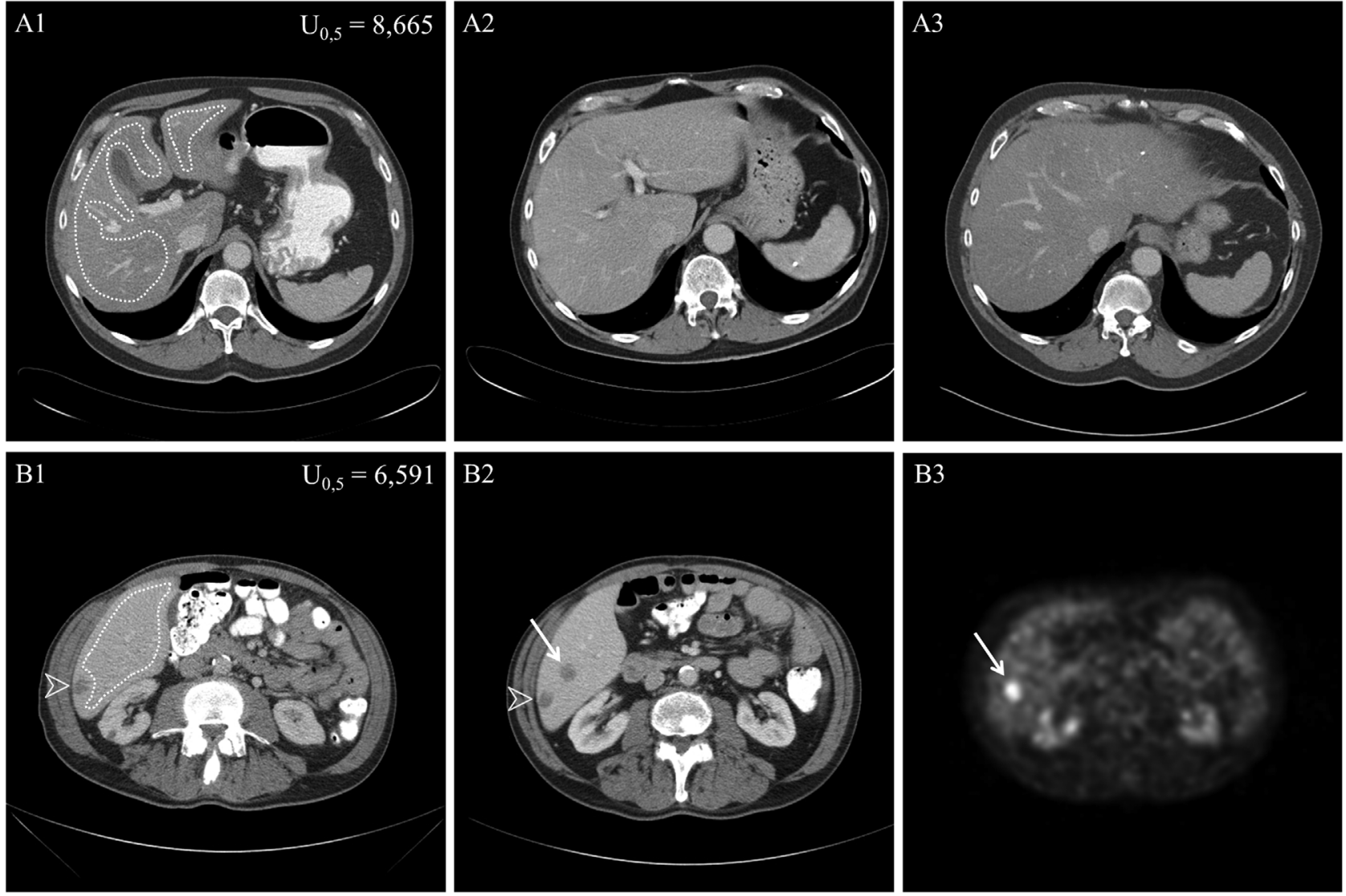

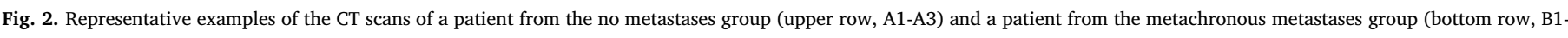

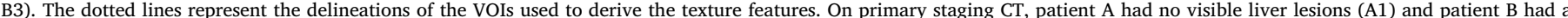

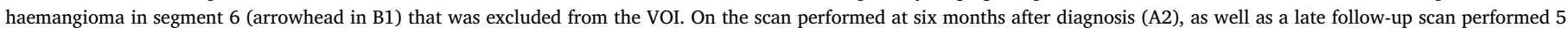

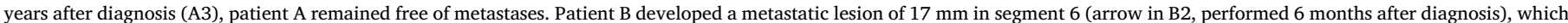

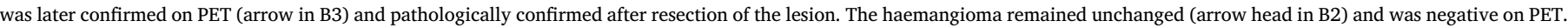

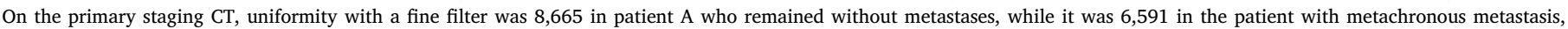
illustrating that lower uniformity may be used as a marker to predict patients at risk of developing early metastatic disease. 

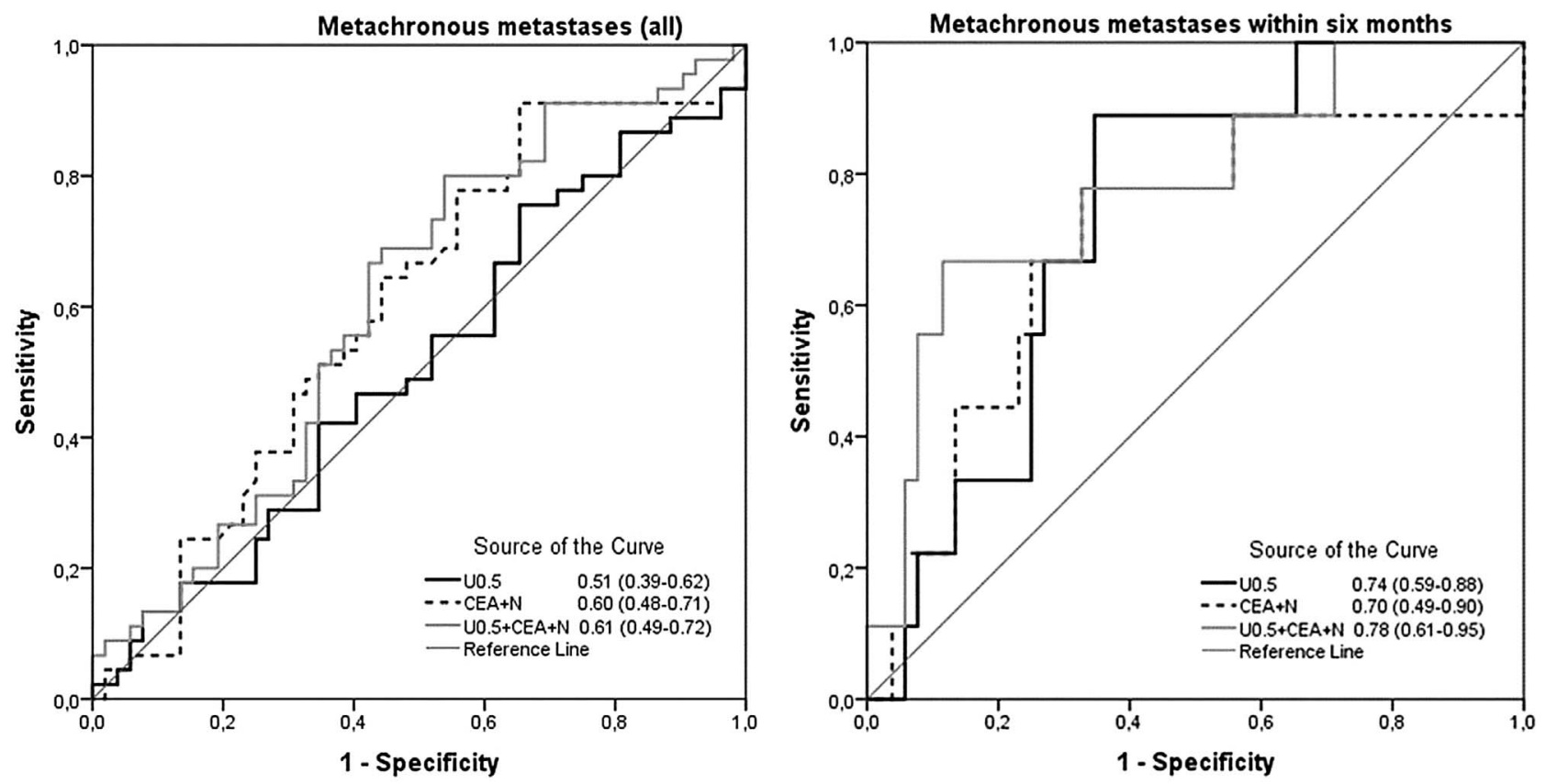

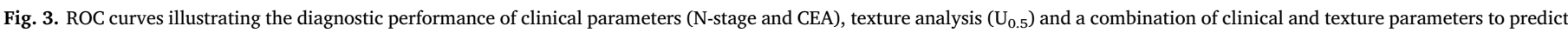
patients at risk to develop metachronous disease for the whole patient group (left) and for the subgroup of patients who developed metastases within six months.

statistically significant $(P=0.08)$. From a clinical point of view, however, these very early metastases are more considered occult synchronous metastases, that were probably already present but too small to detect by visual assessment on CT at the time of primary staging. Moreover, one could wonder whether these lesions might have been detected if patients would have been imaged with MRI instead of CT, given the known superiority of MRI over CT for the detection of small liver metastases [23,24]. For the prediction of 'true' metachronous metastases occurring at later stage, texture analyses were not beneficial. If in these patients changes were already present within the liver at time of primary diagnosis and staging, they were too small to be detected with texture analyses.

Despite the fact that the texture analysis and the delineation were performed in the same way as previous reports, there are some remarkable discrepancies between the findings [10,14,21]. First, in our study the best results were obtained for texture measures using fine image filtration, whereas previous reports found better results for medium to coarse filters $[10,14]$. Fine image filtration highlights very small structures (in the order of $1-2 \mathrm{~mm}$ ), while coarse image filtration highlights larger structures (in the order of $6 \mathrm{~mm}$ ). The size of the structures that can be detected, however, also depends on the chosen slice thickness which influences image noise and intrinsic contrast. Therefore one could expect a correlation between the slice thickness and the optimal image filtration to detect structures of a certain size. Since the above-mentioned studies (including the current) were performed with CT images of varying slice thickness (ranging between 3 and $10 \mathrm{~mm}$ ), this may have contributed to the varying results for different image filtrations between studies. Also, previous studies performed by Ganeshan et al. use a single slice, while our report is based on the average of multiple slices resulting in whole liver analysis $[10,17]$.

Although the exact mechanism is not fully known, changes in liver texture as a result of metastatic involvement have been proposed to be related to changes in liver perfusion or changes on a cellular level caused by the presence of micro metastases [16,18,25] With this in mind, it would be logical to assume that entropy would increase (more heterogeneous/irregular liver structure) and vice versa uniformity would decrease in patients with synchronous metastases and that by extension this effect may also already be observed in patients who later develop metachronous disease. However, as illustrated in Table 2, entropy and uniformity values fluctuated inconsistently between the different subgroups of patients with synchronous, early, intermediate and late metastases for reasons not well understood. Previous studies also found conflicting results; some indeed reported higher entropy and corresponding lower uniformity in patients with metastases [10,14], while others reported the opposite effect [9]. Further research is therefore needed to understand the complex relations between texture parameters and underlying biological tissue characteristics in these different settings.

Apart from biologic tissue properties, there are also several other factors that may influence texture measures, amongst which the image acquisition parameters. For example, Bezy-Wendling et al. reported that texture values are highly dependent on the slice thickness of the CT images [26]. As described above, slice thickness varies considerably between published reports. Moreover, Ganeshan et al. reported that timing of acquisition after contrast injection has a considerable effect on entropy and uniformity values (even with slight variations in timing) with typically an increase in entropy and decrease in uniformity over time $[9,27]$. Since contrast timing differs between studies (some used non-enhanced CT and others contrast-enhanced CT with various contrast timings) and even within studies, this will likely also have contributed to the variations in results $[9,17,25,28]$. Other factors such as contrast volume, $\mathrm{kVp}$ and slice collimation may also affect results, although effects of these items have so far not been specifically addressed in literature. We tried to compensate for such effects by performing a multilevel analysis per CT scanner used in our study (thereby aiming to take into account hardware, software and acquisition protocol related variation between scanners and centres). However we cannot fully rule out potential confounding effects and more detailed research on the precise effects of acquisition-related factors is therefore urgently needed. All together these issues make it difficult to perform multicentre texture research and ultimately generalize and translate findings to daily use. These issues are also part of the reason why texture analysis is not yet ready for use in clinical practice. Before texture features may truly be used as clinical imaging biomarkers, further standardisation of acquisition- and technical parameters is thus required. Moreover, discrimination threshold values will need to be defined and properly validated. 
There are some other limitations to our study design. First, because we aimed to obtain three equally sized patient cohorts, patients (particularly in the metachronous group, being the least prevalent outcome) are non-consecutive. Second, VOI's were drawn by a single observer, which does not account for potential interobserver variations (though we believe that for whole-liver analysis this effect will probably be limited). Finally, slice thickness was relatively large $(5 \mathrm{~mm})$ and in one of the three centres the slice thickness was reconstructed from an original slice thickness of $3 \mathrm{~mm}$, which may have affected image noise. Finally, as described above, for our study we only looked at CT, as this still represents the most routinely used modality for staging CRC in daily practice. Routine staging with MRI was not performed. For future studies it will be worthwhile to look at the potential added value of MRI in this regard.

In conclusion it is our experience that it is technically challenging to analyse CT-texture in a multicentre setting and fully take into account potential confounding factors such as differences in CT acquisition protocols. Apart from these challenges, our results suggest that CTtexture analysis may have promise as an adjunct to known clinical risk factors such as N-stage and CEA to predict which patients that are at risk of developing liver metastases early ( $\leq 6$ months) after initial diagnosis, but is not robust enough to identify patients at risk of developing metastases at later stage, i.e. within 1-2 years.

\section{Funding}

This research did not receive any specific grant from funding agencies in the public, commercial, or not-for-profit sectors.

\section{Acknowledgement}

No additional individuals provided help during the research.

\section{Appendix A. Image filtration}

Texture analysis consists of two main stages: (a) image filtration and (b) quantification of texture.

\section{(A.1) Image filtration}

For image filtration, a Laplacian of Gaussian (LoG) band-pass filter was chosen. Before applying the filter, regions outside the VOI were set to the average value of the pixels inside the VOI, to prevent non-target regions to influence the analysis. The LoG filter is mathematically defined as $\Delta \mathrm{G}(\mathrm{x}, \mathrm{y})$, with $\Delta$ the Laplace operator and $\mathrm{G}(\mathrm{x}, \mathrm{y})$ a two-dimensional Gaussian. We have chosen to normalize the Gaussian as follows:

$G(x, y)=-e^{-\left(x^{2}+y^{2}\right) /\left(2 \sigma^{2}\right)}$

which results in a LoG filter that is described by

$\Delta G=\nabla \cdot \nabla G=\frac{4}{2 \sigma^{2}}\left(1-\frac{x^{2}+y^{2}}{2 \sigma^{2}}\right) e^{-\left(x^{2}+y^{2}\right) /\left(2 \sigma^{2}\right)}$

We applied the filter in the Fourier space, as this is less computationally demanding. To calculate the convolution of the data and our filter, we multiplied their Fourier transforms in Fourier space. This has the additional benefit of reducing the discretization errors, as the filter has a larger width in Fourier space. The Fourier transform of the filter is given by

$F(\Delta G)=2 \pi \sigma^{2} 4 \pi^{2}\left(k_{x}{ }^{2}+k_{y}{ }^{2}\right) e^{-\pi^{2} 2 \sigma^{2}\left(k_{x}{ }^{2}+k_{y}{ }^{2}\right)}$,

with $\mathrm{k}_{\mathrm{x}}$ and $\mathrm{k}_{\mathrm{y}}$ in the range [-0.5,0.5]. The LoG-filter was applied for a range of $\sigma$ values. The width of the filters in pixels (at the reference resolution of $0.84 \mathrm{~mm}$ ) and $\mathrm{mm}$ is shown in Table 1A. For each CT scan with a resolution different than 0.84 mm, the value of $\sigma$ was modified to keep the physical size (in $\mathrm{mm}$ ) of the filter constant. We defined the width of the filter as the distance between the zero crossings (see Fig. 1A). These occur for $x^{2}+y^{2}=2 \sigma^{2}$, so the total width of the filter is given by $2 \sqrt{2} \sigma$.

\section{(A.4) Quantification of texture}

For image quantification of texture, mean grey-level intensity, entropy and uniformity were used. These parameters were calculated from a histogram of the points within the VOI with a bin size of $1 \mathrm{HU}$. From the histogram, the parameters were calculated using:

mean $=\sum_{i=1}^{M} f(i) \cdot h(i)$,

entropy $=-\sum_{i=1}^{M} f(i) \cdot \log _{2}(f(i))$,

uniformity $=1000 \cdot \sum_{i=1}^{M} f(i)^{2}$,

where $\mathrm{M}$ is the number of bins in the histogram, $f(i)$ the fraction of the voxels in the vOI in bin number $i$ of the histogram and $h(i)$ the HU value of bin

Table 1A

Filter widths for different values of $\sigma$

\begin{tabular}{lll}
\hline$\sigma$ [pixels] & Full width [pixels] & Full width [mm] \\
\hline 0.5 & 1.4 & 1.2 \\
1.5 & 4.2 & 3.6 \\
2.5 & 7.0 & 5.9 \\
\hline
\end{tabular}


$i$. The entropy gives an indication of the width of the histogram and the uniformity of the narrowness.

\section{References}

[1] R.L. Siegel, K.D. Miller, A. Jemal, Cancer statistics, 2015, CA. Cancer J. Clin. 65 (1) (2015) 5-29.

[2] S. Manfredi, C. Lepage, C. Hatem, O. Coatmeur, J. Faivre, A.M. Bouvier, Epidemiology and management of liver metastases from colorectal cancer, Ann. Surg. 244 (2) (2006) 254-259.

[3] P. Landreau, A. Drouillard, G. Launoy, P. Ortega-Deballon, V. Jooste, C. Lepage, et al., Incidence and survival in late liver metastases of colorectal cancer, J. Gastroenterol. Hepatol. 30 (1) (2015) 82-85.

[4] A.E. van der Pool, R.A. Damhuis, J.N. Ijzermans, J.H. de Wilt, A.M. Eggermont, R. Kranse, et al., Trends in incidence, treatment and survival of patients with stage IV colorectal cancer: a population-based series, Colorectal Dis. 14 (1) (2012) 56-61.

[5] L.G. van der Geest, J. Lam-Boer, M. Koopman, C. Verhoef, M.A. Elferink, J.H. de Wilt, Nationwide trends in incidence, treatment and survival of colorectal cancer patients with synchronous metastases, Clin. Exp. Metastasis 32 (5) (2015) 457-465.

[6] G.Y. Locker, S. Hamilton, J. Harris, J.M. Jessup, N. Kemeny, J.S. Macdonald, et al., ASCO 2006 update of recommendations for the use of tumor markers in gastrointestinal cancer, J. Clin. Oncol. 24 (33) (2006) 5313-5327.

[7] G.B. Secco, R. Fardelli, D. Gianquinto, P. Bonfante, E. Baldi, E. Campora, Factors influencing incidence and extension of metachronous liver metastases of colorectal adenocarcinoma: a multivariate analysis, Hepatogastroenterology 44 (16) (1997) 1057-1062.

[8] S.C. Chuang, Y.C. Su, C.Y. Lu, H.T. Hsu, L.C. Sun, Y.L. Shih, et al. Risk factors for the development of metachronous liver metastasis in colorectal cancer patients after curative resection, World J. Surg. 35 (2) (2011) 424-429.

[9] B. Ganeshan, K.A. Miles, R.C. Young, C.R. Chatwin, Hepatic entropy and uniformity: additional parameters that can potentially increase the effectiveness of contrast enhancement during abdominal CT, Clin. Radiol. 62 (8) (2007) 761-768.

[10] B. Ganeshan, K.A. Miles, R.C. Young, C.R. Chatwin, Texture analysis in non-contrast enhanced CT: impact of malignancy on texture in apparently disease-free areas of the liver, Eur. J. Radiol. 70 (1) (2009) 101-110.

[11] F. Davnall, C.S. Yip, G. Ljungqvist, M. Selmi, F. Ng, B. Sanghera, et al., Assessment of tumor heterogeneity: an emerging imaging tool for clinical practice? Insights Imaging 3 (6) (2012) 573-589.

[12] B. Ganeshan, K.A. Miles, Quantifying tumour heterogeneity with CT, Cancer Imaging 13 (2013) 140-149.

[13] C. Chen, L. Pau, P. Wang, The handbook of pattern recognition and computer vision, World Scienti. (1998) 207-248.

[14] S.X. Rao, D.M. Lambregts, R.S. Schnerr, W. van Ommen, T.J. van Nijnatten, M.H. Martens, et al., Whole-liver CT texture analysis in colorectal cancer: does the presence of liver metastases affect the texture of the remaining liver? United Eur.
Gastroenterol. J. 2 (6) (2014) 530-538.

[15] J.B. Kruskal, P. Thomas, R.A. Kane, S.N. Goldberg, Hepatic perfusion changes in mice livers with developing colorectal cancer metastases, Radiology 231 (2) (2004) $482-490$.

[16] C. Cuenod, I. Leconte, N. Siauve, A. Resten, C. Dromain, B. Poulet, et al., Early changes in liver perfusion caused by occult metastases in rats: detection with quantitative CT, Radiology 218 (2) (2001) 556-561.

[17] B. Ganeshan, K.A. Miles, R.C. Young, C.R. Chatwin, Hepatic enhancement in colorectal cancer: texture analysis correlates with hepatic hemodynamics and patient survival, Acad. Radiol. 14 (12) (2007) 1520-1530.

[18] E. Leen, The detection of occult liver metastases of colorectal carcinoma, J. Hepatobiliary. Pancreat. Surg. 6 (1) (1999) 7-15.

[19] M. Nanko, H. Shimada, H. Yamaoka, K. Tanaka, H. Masui, K. Matsuo, et al., Micrometastatic colorectal cancer lesions in the liver, Surg. Today 28 (7) (1998) 707-713.

[20] C. Rorden, H.O. Karnath, L. Bonilha, Improving lesion-symptom mapping, J. Cogn. Neurosci. 19 (7) (2007) 1081-1088.

[21] S.X. Rao, D.M. Lambregts, R.S. Schnerr, R.C. Beckers, M. Maas, F. Albarello, et al., CT texture analysis in colorectal liver metastases: a better way than size and volume measurements to assess response to chemotherapy? United Eur. Gastroenterol. J. 4 (2) (2016) 257-263.

[22] E.R. DeLong, D.M. DeLong, D.L. Clarke-Pearson, Comparing the areas under two or more correlated receiver operating characteristic curves: a nonparametric approach, Biometrics 44 (3) (1988) 837-845.

[23] S. Bipat, M.S. van Leeuwen, J.N. Ijzermans, P.M. Bossuyt, J.W. Greve, J. Stoker, Imaging and treatment of patients with colorectal liver metastases in the Netherlands: a survey, Netherlands J. Med. 64 (5) (2006) 147-151.

[24] M.C. Niekel, S. Bipat, J. Stoker, Diagnostic imaging of colorectal liver metastases with CT, MR, imaging, FDG PET, and/or FDG PET/CT: a meta-analysis of prospective studies including patients who have not previously undergone treatment, Radiology 257 (3) (2010) 674-684.

[25] B. Ganeshan, K.A. Miles, R.C. Young, C.R. Chatwin, In search of biologic correlates for liver texture on portal-phase CT, Acad. Radiol. 14 (9) (2007) 1058-1068.

[26] J. Bezy-Wendling, M. Kretowski, Y. Rolland, W. Le Bidon, Toward a better understanding of texture in vascular CT scan simulated images, IEEE Trans. Biomed. Eng. 48 (1) (2001) 120-124.

[27] B. Ganeshan, K. Burnand, R. Young, C. Chatwin, K. Miles, Dynamic contrastenhanced texture analysis of the liver: initial assessment in colorectal cancer, Invest. Radiol. 46 (3) (2011) 160-168.

[28] K.A. Miles, B. Ganeshan, M.R. Griffiths, R.C. Young, C.R. Chatwin, Colorectal cancer: texture analysis of portal phase hepatic CT images as a potential marker of survival, Radiology 250 (2) (2009) 444-452. 